\title{
On the Diversity of Partial Relaying Cooperation with Relay Selection in Finite-SNR Regime
}

\author{
Thang X. Vu, Symeon Chatzinotas, and Bjorn Ottersten \\ Interdisciplinary Centre for Security, Reliability and Trust (SnT), \\ University of Luxembourg. Email: \{thang.vu, symeon.chatzinotas, bjorn.ottersten\}@uni.lu
}

\begin{abstract}
This work studies the performance of a cooperative network which consists of two channel-coded sources, multiple relays, and one destination. Due to the spectral efficiency constraint, we assume that a single time slot is dedicated to relaying. Conventional network-coded based cooperation (NCC) selects the best relay which uses network coding to serve the two sources simultaneously. It is shown that NCC, however, only achieves diversity of order two regardless of the number of available relays and the channel code. In this paper, we propose a novel partial relaying based cooperation (PARC) scheme to improve the system diversity in the finite signal-to-noise ratio (SNR) regime. Firstly, closed-form expressions for the system bit error rate (BER) and diversity order of PARC are derived as a function of the operating SNR value and the minimum distance of the channel code. Secondly, we analytically show that the proposed PARC achieves full diversity order in the finite SNR regime, given that an appropriate channel code is used. Finally, numerical results verify our analysis and demonstrate a large SNR gain of PARC over NCC in the SNR region of interest.
\end{abstract}

Index terms- Cooperative diversity, relay selection, partial relaying, channel coding.

\section{INTRODUCTION}

Cooperation among nodes is an effective technique to widen the coverage and improve the performance of wireless networks in both terms of signal-to-noise ratio (SNR) and diversity gains [1]. Such improvement, however, usually comes at the price of an additional orthogonal channel, resulting in a reduced spectral efficiency, which can be significant in multiple-relay networks. In order to reduce this loss, opportunistic relay selection (RS) has been proposed to select the best relay for cooperation [2]. It has been shown that RS achieves full diversity order for single-source multiple-relay networks and outperforms other relaying schemes in terms of SNR gain and effective capacity [3].

Recently, there has been much interest on combining RS together with network coding to further improve the spectral efficiency. It is shown via outage probability analysis that the use of RS in a two-way relay channel (TWRC) could achieve full diversity order and a significant SNR gain [4][6]. While research on RS in TWRC is readily available in the literature, research on RS in unidirectional relay networks is still limited. This problem is first considered in [7], which analyzes diversity multiplexing tradeoff (DMT) and shows that full diversity order is achieved. However, [7] relies on an unrealistic assumption that unintended packets are available

This work is financially supported in part by Luxembourg National Research Fund Core program under the project code I2R-SIG-PFN-13SEMI. at all destinations. A generalized DMT analysis is presented in [8]. Likewise, [8] relies on an optimistic assumption that the selected channels are mutually independent, which is infeasible because these channels belong to an ordered SNR sequence, and thus are highly correlated [5]. By removing such unrealistic assumption, it is shown in our previous work that NCC fails to achieve full underlying diversity gain [11] regardless of how many available relays are. It is worthy noting that the above-mentioned works study the system diversity via the upper-bound limit of the BER or outage probability (OP) in the absence of channel coding, which might be in contrast to practical scenarios where nodes are usually protected by some forward error correction codes.

In this paper, we investigate the performance of cooperative networks under practical scenarios, i.e., the transmitted signals are protected by convolutional codes (CC). Due to the constraint on spectral efficiency and processing delay, it is assumed that only one time slot is dedicated to cooperation. The best RS is employed to effectively exploit the spatial diversity [2]. At the destination, the cooperative maximal ratio combining (C-MRC) detector [9] is used prior to channel decoding to mitigate error propagation. We would highlight that C-MRC is a suboptimal detector but provides full diversity gain and close performance to the maximum likelihood (ML) receiver [10].

The contributions of this paper are as follows:

- Firstly, we propose a partial relaying based cooperation in Section II to select the two best relays for cooperation, each helping one source independently. The proposed PARC is different from NCC, where the best relay is chosen based on the equivalent network-coded channel. Compared to [13], [14], our proposed scheme has two main differences: i) we analyse the system via BER, whereas these papers study the system OP, which is fundamentally different from our setting (we can obtain the actual BER for arbitrary SNR value); and ii) we investigate RS to improve the spectral efficiency, while these papers consider single-relay networks.

- Secondly, insightful theoretical analysis is provided for PARC in the finite-SNR regime in Section III. In particular, closed-form expressions for the BER and instantaneous diversity order ${ }^{1}$ are derived, which reveal the

\footnotetext{
${ }^{1}$ Instantaneous diversity order is measured as the slope of the BER curve in log-log scale, which allows to study the system behavior at arbitrary SNR value. This definition coincides with the conventional diversity definition in the high SNR regime [15]
} 
dependency of the instantaneous diversity order on the operating SNR value and the minimum distance of the channel code.

- Finally, numerical results in Section IV demonstrate the effectiveness of our proposed scheme. It is shown via both analytical and simulation results that PARC can achieve full diversity order in the low and medium SNR regime when a suitable CC is used. This result is important since the practical systems usually operate in the finite SNR regime.

\section{Partial Relaying Based Cooperation (PARC)}

The system under consideration consists of two sources denoted by $\mathrm{S}_{1}$ and $\mathrm{S}_{2}, N_{r}$ relays denoted by $\mathrm{R}_{i}$ with $1 \leq i \leq N_{r}$, and one destination denoted by $\mathrm{D}$. Such a scenario can find applications in uplinks cellular mobile systems in which two mobile users send data to the base station and some friendly, idle users who can act as the relays. All nodes are equipped with a half-duplex single antenna. We assume orthogonal block Rayleigh fading channels and perfect time synchronization. As a result, one cooperation period is divided into two phases: broadcast and relaying. In the first phase, two sources consecutively broadcast data to the relays and destination. In the second phase, the selected relay forwards signal to the destination. Demodulate-and-forward relaying protocol [16] is used to minimize the relay's computational complexity. Due to the spectral efficiency constraint and processing time limit, we assume that a single time slot is dedicated to the relaying phase.

Conventional NCC selects the best relay based on the equivalent channel of network-coded symbols, which, unfortunately, only achieves diversity order of two regardless how many available relays are [11]. In this work, we propose PARC which selects two best relays for the cooperation, each helping one source. Since two relays are active in the relaying phase, each relay only occupies half of relaying time slot, as shown in Fig. 1b. Consequently, the selected relay can only forward half of the estimated codeword to the destination. Although forwarding half of the estimated codeword, we will show that a good instantaneous diversity gain is achievable when a suitable channel code is employed.

\section{A. Relay Selection in PARC}

The selection process is executed at the beginning of each block in a distributed manner [2] based on the maxmin criterion that maximizes the worst end-to-end SNR and reduces computational complexity [4]. It is observed that the end-to-end performance of relayed symbols is determined by the weaker between source-relay and relay-destination connections. We thus model a two-hop source-relay-destination link by an equivalent single-hop channel, which is highly accurate for DMF relaying protocol [2]. Let $\gamma_{X Y}=P_{X Y}\left|h_{X Y}\right|^{2} / \sigma^{2}$ with $X \in\left\{\mathrm{S}_{i}, \mathrm{R}_{j}\right\}, Y \in\left\{\mathrm{R}_{j}, D\right\}$ denote the instantaneous channel gain of the link $\mathrm{X} \rightarrow \mathrm{Y}$, where $h_{X Y}$ is the channel coefficient between $\mathrm{X}$ and $\mathrm{Y}, P_{X Y}$ is the received power including the path loss, and $\sigma^{2}$ is the noise power. Then the two-hop $\mathrm{S}_{i} \rightarrow \mathrm{R}_{j} \rightarrow \mathrm{D}$ channel is well modeled by

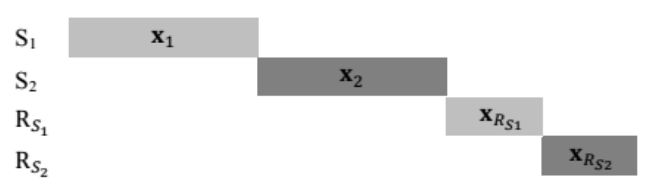

(a)

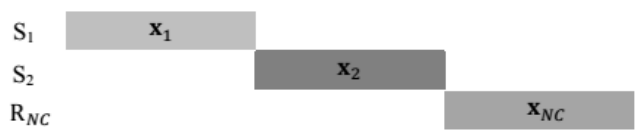

(b)

Fig. 1: Time allocation for the proposed PARC (a) compared with NCC (b).

$\gamma_{i j}=\min \left\{\gamma_{S_{i} R_{j}}, \gamma_{R_{j} D}\right\}, 1 \leq j \leq N_{r}$. Since both $\gamma_{S_{i} R_{j}}$ and $\gamma_{R_{j} D}$ are exponential random variables with mean $\bar{\gamma}_{S_{i} R_{j}}$ and

$\bar{\gamma}_{R_{j} D}$, respectively, it is straightforward to show that $\gamma_{i j}$ is also an exponential random variable with mean $\bar{\gamma}_{i j}=\frac{\bar{\gamma}_{S_{i} R_{j}} \bar{\gamma}_{R_{j} D}}{\bar{\gamma}_{S_{i} R_{j}}+\bar{\gamma}_{R_{j} D}}$. The best relay for source $\mathrm{S}_{i}, i=1,2$, denoted by $\mathrm{R}_{i}^{\star}$, is selected to achieve the largest equivalent channel as follows:

$$
\mathrm{R}_{i}^{\star}=\arg \max _{R_{j}} \gamma_{i j}, 1 \leq j \leq N_{r} .
$$

The equivalent channel of the selected relay, $\gamma_{R_{i}^{\star}}$, is given by:

$$
\gamma_{R_{i}^{\star}}=\max \left\{\gamma_{i 1}, \ldots, \gamma_{i N_{r}}\right\} \text {. }
$$

By using the Max function [19], the PDF of $\gamma_{R_{i}^{\star}}$ is given in a shortened form as follows:

$f_{\gamma_{R_{i}^{\star}}}(\gamma)=\sum_{j=1}^{N_{r}}\left((-1)^{j-1} \sum_{\substack{n_{1}=1, \ldots, n_{j}=1 \\ n_{1} \neq \cdots \neq n_{j}}}^{N_{r}} \frac{1}{\bar{\gamma}_{R_{i}^{\star}, j}} \exp \left(-\frac{\gamma}{\bar{\gamma}_{R_{i}^{\star}, j}}\right)\right)$,

where $\frac{1}{\bar{\gamma}_{R_{i}^{\star}, j}}=\sum_{k=n_{1}}^{n_{j}}\left(\frac{1}{\bar{\gamma}_{S_{i} R_{k}}}+\frac{1}{\bar{\gamma}_{R_{k} D}}\right)$.

The moment-generating function (MGF) of $\gamma_{R_{i}^{\star}}$ is given by:

$$
\Psi_{\gamma_{R_{i}^{\star}}}(s)=\sum_{j=1}^{N_{r}}\left((-1)^{j-1} \sum_{\begin{array}{c}
n_{1}=1, \ldots, n_{j}=1 \\
n_{1} \neq \cdots \neq n_{j}
\end{array}}^{N_{r}} \frac{1}{1+\bar{\gamma}_{R_{i}^{\star}, j} s}\right) .
$$

We would highlight that the relay selection process in PARC is performed for each source separately, which is different from NCC.

\section{B. Demodulate-and-Forward Relaying}

Once the RS is completed, the sources begin sending data to the destination with help of the selected relays. Denote $\mathbf{c}_{i}$ and $\mathbf{x}_{i}$ as an information codeword and the corresponding modulated signal transmitted by source $S_{i}$. At the end of the broadcast phase, the received signals at the destination and selected relays are given as

$$
\left\{\begin{array}{l}
\mathbf{y}_{S_{i} R_{i}^{\star}}=\sqrt{P_{S_{i} R_{i}^{\star}}} h_{S_{i} R_{i}^{\star}} \mathbf{x}_{i}+\mathbf{n}_{S_{i} R_{i}^{\star}}, i=1,2, \\
\mathbf{y}_{S_{i} D}=\sqrt{P_{S_{i} D}} h_{S_{i} D} \mathbf{x}_{i}+\mathbf{n}_{S_{i} D}, i=1,2 .
\end{array}\right.
$$

The selected relay estimates the source coded symbols and forwards them to the destination. In particular, the selected 
relay $\mathrm{R}_{i}^{\star}, i=1,2$, uses half of the relaying time slot to forward half of the codeword $\mathbf{c}_{i}$ to the destination. More specifically, $\mathrm{R}_{i}^{\star}$ first estimates $L=N / 2$ (without loss of generality, assuming $N$ is even) coded symbols to form a punctured estimation $\hat{\mathbf{c}}_{R_{i}^{\star}}=\left\{\hat{c}_{R_{i}^{\star}, l}\right\}_{l \in \Theta_{i}}$, where $\Theta_{i}=\left\{k_{1}, k_{2}, \ldots, k_{L}\right\}$ is the set of the symbol indexes which are helped by $\mathrm{R}_{i}^{\star}$. The index set $\Theta_{i}$ is determined randomly ${ }^{2}$. The coded symbols at the relay are estimated by the ML detector as follows:

$$
\hat{c}_{R_{i}^{\star}, l}=\arg \min _{c_{i, k_{l}} \in\{0,1\}}\left\{\left|y_{S_{i} R_{i}^{\star}, k_{l}}-\sqrt{P_{S_{i} R_{i}^{\star}}} h_{S_{i} R_{i}^{\star}} x_{i, k_{l}}\right|^{2}\right\},
$$

$\forall k_{l} \in \Theta_{i}$, where $x_{i, k_{l}}$ being the corresponding modulated symbol of $c_{i, k_{l}}$. Next, $\mathrm{R}_{i}^{\star}$ modulates $\hat{\mathbf{c}}_{R_{i}^{\star}}$ into the modulated signal $\hat{\mathbf{x}}_{R_{i}^{\star}}$ and then forwards it along with $\Theta_{i}$ to the destination. The cost for conveying $\Theta_{i}$ is negligible since it can send, e.g., the seed of the random interleaver, to the destination.

\section{Decoding at the Destination}

The received signal at the destination from the selected relay is given as

$$
\mathbf{y}_{R_{i}^{\star} D}=\sqrt{P_{R_{i}^{\star} D}} h_{R_{i}^{\star} D} \hat{\mathbf{x}}_{R_{i}^{\star}}+\mathbf{n}_{R_{i}^{\star} D}, i=1,2,
$$

where $h_{R_{i}^{\star} D}$ is the channel coefficient from $\mathrm{R}_{i}^{\star} \rightarrow \mathrm{D}$, and $\mathbf{n}_{R_{i}^{\star} D}$ is a noise vector whose components are Gaussian random variable with zero mean and variance $\sigma^{2}$.

After receiving two signals from the source and the selected relay, the destination starts the decoding process with two consecutive steps: demodulating and decoding. We assume that the CSI of all channels, i.e., $\mathrm{S}_{i} \rightarrow \mathrm{D}, \mathrm{S}_{i} \rightarrow \mathrm{R}_{i}^{\star}$, and $\mathrm{R}_{i}^{\star} \rightarrow \mathrm{D}$ channels, are available at the destination. The CSIs can be obtained via pilot training. The destination first applies the C-MRC detector [9] to demodulate the coded bits for source $\mathrm{S}_{i}, i=1,2$, as $\hat{c}_{i, k}=\arg \min _{c_{i, k} \in\{0,1\}} \mathcal{M}\left(x_{i, k}\right), 1 \leq k \leq N$, where $\mathcal{M}\left(x_{i, k}\right)=\left|y_{S_{i} D, k}-\sqrt{P_{S_{i} D}} h_{S_{i} D} x_{i, k}\right|^{2}$ if $k \notin \Theta_{i}$; otherwise

$$
\begin{aligned}
\mathcal{M}\left(x_{i, k}\right)= & \left|y_{S_{i} D, k}-\sqrt{P_{S_{i} D}} h_{S_{i} D} x_{i, k}\right|^{2} \\
& +\lambda_{R_{i}^{\star}}\left|y_{R_{i}^{\star} D, k}-\sqrt{P_{R_{i}^{\star} D}} h_{R_{i}^{\star} D} \hat{x}_{R_{i}^{\star}, k}\right|^{2} .
\end{aligned}
$$

In (4), $\lambda_{R_{i}^{\star}}$ is the parameter of the C-MRC detector which is computed as $\lambda_{R_{i}^{\star}} \triangleq \frac{\min \left(\gamma_{\left.S_{i} R_{i}^{\star}, \gamma_{R_{i}^{\star} D}\right)}\right.}{\gamma_{R_{i}^{\star} D}}$. The C-MRC detector then computes log-likelihood ratio values of the coded bits and sends them to the channel decoder. Finally, the channel decoder applies the BCJR algorithm [18] to decode the transmitted data.

Remark 1: In our protocol, the selected relay always forwards the estimated symbols to the destination. Fortunately, possible decoding errors in $\hat{c}_{R_{i}^{\star}, l}$, hence error propagation, is effectively mitigated by C-MRC via $\lambda_{R_{i}^{\star}}$. For example, if the source-relay channel is too noisy, i.e., $\gamma_{S_{i} R_{i}^{\star}}$ is too small, it is highly probable that $\mathrm{R}_{i}^{\star}$ decodes with errors. In this case, however, $\lambda_{R_{i}^{\star}}$ is small and the contribution of the relayed signal is negligible.

\footnotetext{
${ }^{2}$ Other selection of $\Theta_{i}$, e.g., optimal index set, can be employed, but are beyond the scope of this paper.
}

\section{Performance Analysis for Partial Relaying BASED COOPERATION}

In this section, we analyze the BER and diversity order of PARC by using the equivalent channel model. Since PARC is symmetric, the analysis for two sources is similar. For ease of presentation, we drop the source subscript in this section. After two phases, the destination receives two signals from the source $S$ and the selected relay $R^{\star}$. The combined signal at the C-MRC detector's output can be classified into two groups: the first group consists of symbols which are helped by the selected relay, and the second group includes the rest of the symbols which are not relayed. In other words, the received signal at the destination can be seen as an output of a block fading channel with 2 blocks: one block consisting of the first $N / 2$ symbols which only see channel $\gamma_{S D}$, and the other one contains the other $N / 2$ symbols which see both channel $\gamma_{S D}$ and $\gamma_{R^{\star}}$.

\section{A. Bit Error Rate Analysis}

Let $\overline{\operatorname{PEP}}(d)$ be the unconditioned pair-wise error probability (UPEP), which is the probability that the destination correctly decodes a codeword with Hamming weight $d$ (number of non-zero coded bits in $\mathbf{c}$ at the source) when the allzero codeword was transmitted. The BER of PARC is upperbounded as follows [20]:

$$
\mathrm{BER} \leq \sum_{d=f}^{N} w(d) \overline{\operatorname{PEP}}(d),
$$

where $f$ is the minimum distance of the channel code and $w(d)$ are the input weights (number of non-zero information bits) which are computed directly from the structure of the code. The $\overline{\operatorname{PEP}}(d)$ is the expectation over the channel fading coefficients of the conditioned pair-wise error probability (CPEP), $\operatorname{PEP}(d)$, as $\overline{\operatorname{PEP}}(d)=\mathbb{E}\{\operatorname{PEP}(d)\}$. Obviously, $\operatorname{PEP}(d)$ depends on the channel fading coefficients and the weight pattern $\mathbf{D}_{d}=\left\{d_{1}, d_{2}\right\}, d_{1}+d_{2}=d$, which specifies how the $d$ non-zero coded bits are distributed within the two blocks $\left(\gamma_{S D}\right.$ and $\left.\gamma_{S D}+\gamma_{R^{\star}}\right)$. Because $d$ non-zero coded bits uniformly locate in the two blocks, the CPEP can be further analyzed as follows:

$$
\operatorname{PEP}(d)=\sum_{\mathbf{D}_{d}} \operatorname{PEP}\left(d \mid \mathbf{D}_{d}\right) p\left(\mathbf{D}_{d}\right),
$$

where $p\left(\mathbf{D}_{d}\right)$ is the probability of pattern $\mathbf{D}_{d}$, which is computed by combinatoric computation as

$$
p\left(\mathbf{D}_{d}\right)=\frac{\mathcal{C}_{d_{1}}^{N / 2} \times \mathcal{C}_{d_{2}}^{N / 2}}{\mathcal{C}_{d}^{N}}
$$

where $\mathcal{C}_{k}^{n}=\frac{n !}{(n-k) ! \times k !}$.

Substituting (6) into $\overline{\operatorname{PEP}}(d)$ we obtain:

$$
\overline{\operatorname{PEP}}(d)=\sum_{\mathbf{D}_{d}} \underbrace{\mathbb{E}\left\{\operatorname{PEP}\left(d \mid \mathbf{D}_{d}\right)\right\}}_{\overline{\operatorname{PEP}}\left(d \mid \mathbf{D}_{d}\right)} p\left(\mathbf{D}_{d}\right) .
$$

Given the pattern $\mathbf{D}_{d}=\left\{d_{1}, d_{2}\right\}$, there are $d_{1}$ non-zero coded bits carried by block $\gamma_{S D}$ and $d_{2}$ non-zero coded bits carried 
by block $\gamma_{S D}+\gamma_{R^{\star}}$. As a result, the CPEP PEP $\operatorname{PA}_{\mathrm{PA}}\left(d \mid \mathbf{D}_{d}\right)$ is calculated, using similar techniques in [21], as follows:

$$
\operatorname{PEP}\left(d \mid \mathbf{D}_{d}\right)=Q\left(\sqrt{2 \gamma_{\Sigma}}\right)
$$

where $\gamma_{\Sigma}=d_{1} \gamma_{S D}+d_{2}\left(\gamma_{S D}+\gamma_{R^{\star}}\right)=d \gamma_{S D}+d_{2} \gamma_{R^{\star}}$.

Taking into account the independence between $\gamma_{S D}$ and $\gamma_{R^{\star}}$, we have $\Psi_{\gamma_{\Sigma}}(s)=\Psi_{\gamma_{S D}}(d s) \times \Psi_{\gamma_{R^{\star}}}\left(d_{2} s\right)$.

Theorem 1: Given the weight pattern $\mathbf{D}_{d}=\left\{d_{1}, d_{2}\right\}, d=$ $d_{1}+d_{2}$, the UPEP $\overline{\mathrm{PEP}}\left(d \mid \mathbf{D}_{d}\right)$ of PARC is calculated as

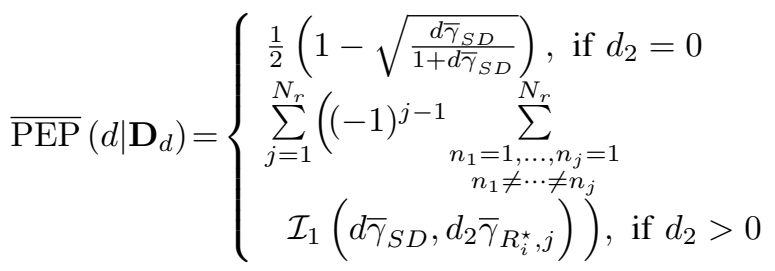

where $\mathcal{I}_{1}(a, b) \triangleq \frac{1}{2}\left(1-\frac{a}{a-b} \sqrt{\frac{a}{a+1}}-\frac{b}{b-a} \sqrt{\frac{b}{b+1}}\right)$ and $\bar{\gamma}_{R_{i}^{\star}, j}$ is given in Section II-A.

Proof: See Appendix A.

Substituting $\overline{\operatorname{PEP}}\left(d \mid \mathbf{D}_{d}\right)$ in Theorem 1 into (8) and (5), we obtain the upper bound for the BER. Note that even though $d$ in (5) can be as large as the codeword's length, i.e., $N$, the BER usually depends on a few first values in fading channels. To give insightful understanding of PARC, we analyze the system diversity order.

\section{B. Diversity Analysis for $\overline{\mathrm{PEP}}\left(d \mid \mathbf{D}_{d}\right)$}

We first analyze the diversity order of the UPEP $\overline{\operatorname{PEP}}\left(d \mid \mathbf{D}_{d}\right)$ for a given weight pattern $\mathbf{D}_{d}$, which determines how the selected relay contributes to the overall system performance.

Theorem 2: Given the weight pattern $\mathbf{D}_{d}=\left\{d_{1}, d_{2}\right\}$ with $d=d_{1}+d_{2}$, the UPEP $\overline{\operatorname{PEP}}\left(d \mid \mathbf{D}_{d}\right)$ in PARC has an exponential equivalent given as follows:

$$
\overline{\operatorname{PEP}}\left(d \mid \mathbf{D}_{d}\right) \propto\left\{\begin{array}{ll}
\bar{\gamma}^{-1}, & \text { if } d_{2}=0 \\
\bar{\gamma}^{-}\left(N_{r}+1\right), & \text { if } d_{2}>0
\end{array},\right.
$$

where $\propto$ denotes the proportional relation.

Proof: See Appendix B.

Theorem 2 states that $\overline{\operatorname{PEP}}\left(d \mid \mathbf{D}_{d}\right)$ can have either diversity order one or diversity order of $N_{r}+1$ depending on the weight pattern $\mathbf{D}_{d}$.

\section{Diversity Analysis of PARC}

This subsection analyzes the diversity order of the proposed PARC scheme. It is observed from (5) that the diversity order of PARC is determined by $\overline{\operatorname{PEP}}(d)$ because the input weight $w(d)$ of the channel code is constant. From (8) we conclude that $\overline{\mathrm{PEP}}(d)$ is a combination of one factor with diversity of order one (corresponding to the pattern $D_{1}=(d, 0)$ ) and one factor with diversity of order $N_{r}+1$. The contribution of the factor with diversity order one is computed from (7) as follows:

$$
p\left(D_{1}\right)=\frac{\mathcal{C}_{d}^{N / 2}}{\mathcal{C}_{d}^{N}}=\prod_{k=0}^{d-1} \frac{N-2 k}{2 N-2 k}
$$

In practices, the codeword length $N$ is usually much larger than $d$, then $p\left(D_{1}\right)$ can be well-approximated as

$$
p\left(D_{1}\right) \simeq\left(\frac{1}{2}\right)^{d} \leq\left(\frac{1}{2}\right)^{f} .
$$

From (10), (5), and Theorem 2, we have

$$
\operatorname{BER}(\bar{\gamma})=K 2^{-f} \bar{\gamma}^{-1}+\bar{\gamma}^{-N_{r}-1}
$$

where $K$ is the normalized constant that depends on the channel code and network topology.

The classical definition of diversity order is defined as the negative exponent of the average BER as a function of SNR in $\log -\log$ scale [15], which visually represents the slope of the BER curve at the high SNR regime. In this paper, since we are interested in the system behavior in the finite-SNR regime, we define the diversity order at an arbitrary (average) SNR $\bar{\gamma}$ as the slope of BER curve in the log-log scale [17]:

$$
\begin{aligned}
\zeta(\bar{\gamma}) & \triangleq-\lim _{\Delta \rightarrow 0} \frac{\log (\operatorname{BER}(\bar{\gamma}+\Delta))-\log (\operatorname{BER}(\bar{\gamma}))}{\log (\bar{\gamma}+\Delta)-\log (\bar{\gamma})} \\
& =-\left.\gamma \frac{\partial \log (\operatorname{BER}(\gamma))}{\partial \gamma}\right|_{\gamma=\bar{\gamma}},
\end{aligned}
$$

which obviously matches the classical definition of diversity when the SNR tends to infinity. Because the diversity order depends on the SNR, we refer to $\zeta(\bar{\gamma})$ as instantaneous diversity order. The key idea behind the definition is that it allows the system behavior to be studied at any SNR value.

Substituting (11) into (12), we obtain the instantaneous diversity order of PARC as follows:

$$
\zeta(\bar{\gamma})=1+\frac{N_{r}}{1+K 2^{-f} \bar{\gamma}^{N_{r}}} .
$$

An important observation from (13) is that the instantaneous diversity order of PARC depends on the operating SNR and the channel code, which provides a criterion design to achieve desirable diversity order in the finite-SNR regime. By choosing a proper channel code whose minimum distance $f$, such as

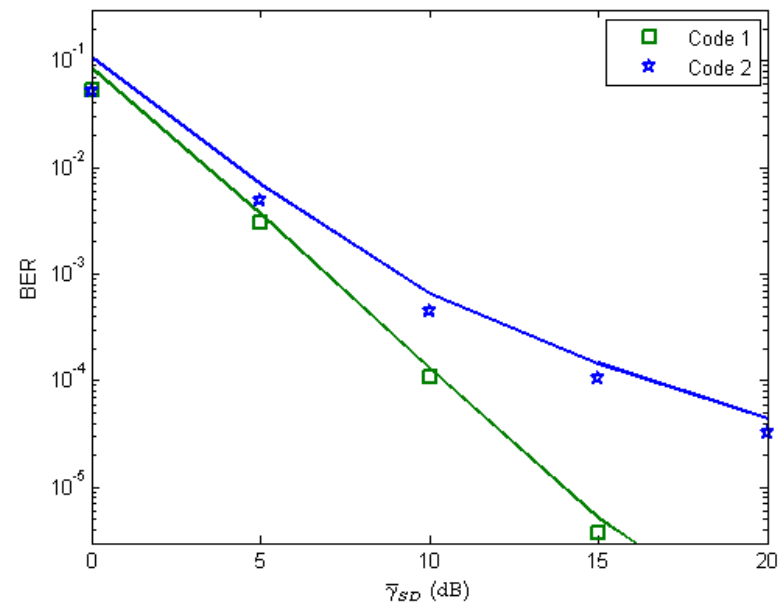

Fig. 2: Validation of the analysis for both PARC. $N_{r}=2$, code 1: [133 165 171], code 2: [5 7 5]. 


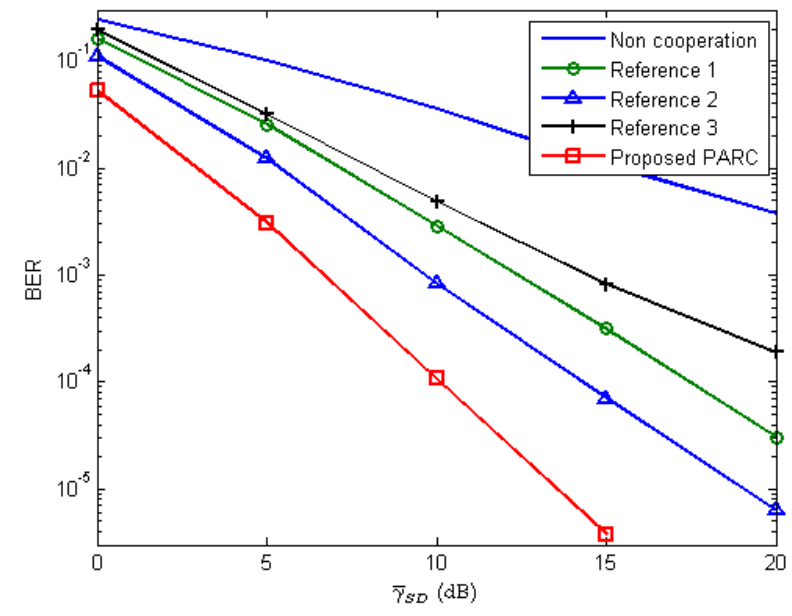

(a) $N_{r}=2$

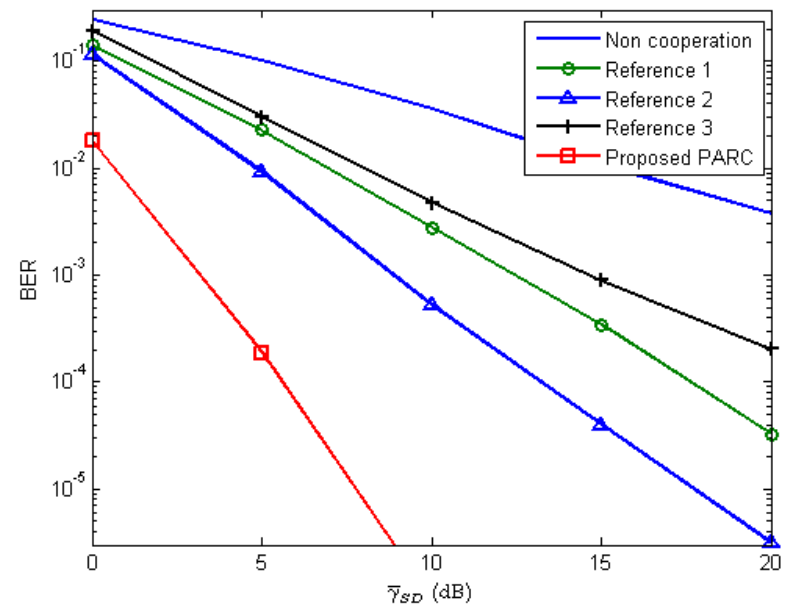

(b) $N_{r}=4$

Fig. 3: Performance comparison between PARC and NCC when the CC [133 165 171] with the minimum distance $f=15$ and the rate $1 / 3$ is used.

$K 2^{-f} \bar{\gamma}_{*}^{N_{r}} \ll 1$, then the PARC achieves full diversity order of $N_{r}+1$ in the SNR region $\left[0, \bar{\gamma}_{*}\right]$. This result is crucial because the operating SNR regime is usually finite in practice.

\section{NumericAl RESULts}

This section presents simulation results to confirm the effectiveness of the proposed PARC. All channels are subject to quasi-static block Rayleigh fading plus AWGN. Since we focus on the diversity order, and the modulation order does not change the system diversity order, BPSK modulation and binary network coding are carried out in simulations. Each data packet consists of 1024 bits. We consider symmetric network, i.e., $\bar{\gamma}_{S_{i} R_{j}}=\bar{\gamma}_{S R}, \bar{\gamma}_{R_{j} D}=\bar{\gamma}_{R D}, \bar{\gamma}_{S_{i} D}=\bar{\gamma}_{S D}, \forall i, j$. The relays are located in the middle between the sources and the destination. The pathloss exponent is equal to 3.5. As the result, the average SNR in source-relay channels and relaydestination channels are $10.5 \mathrm{~dB}$ better than source-destination channels. Note that our analysis holds for arbitrary locations of the relays. The channel code is selected in order to optimize both the minimum distance and distance spectrum in block Rayleigh fading channels [22]. Three reference schemes are presented for comparison. Reference 1 uses relay selection based on the network coding cooperation NCC [11]. Reference 2 is based on fractional repetition coding cooperation [13], [14]. The reference 3 scheme employs fractional repetition coding together with network coding. Note that in the references 2 and 3, all relays are active and share the relaying phase without relay selection.

Figure 2 compares the bounds derived in Theorem 1 with the simulations. The first 6 output weights are used to compute the bounds. It is shown that the derived bounds are consistent with simulation results for different channel codes, which demonstrates the accuracy of our analysis.

Figure 3 compares the performance of PARC with the references. CC [133 165 171] with code rate $1 / 3$ and minimum distance $f=15$ is used. The total number of relays $N_{r}=2$ and $N_{r}=4$ are plotted. The observed performance region is until $\mathrm{BER} \geq 10^{-6}$, which satisfies most practical applications. It is shown in the figure that the proposed PARC achieves an instantaneous diversity order of 3 and 5 (full diversity order) in the observed SNR range for $N_{r}=2$ and $N_{r}=4$, respectively. Such result can be explained from Theorem 1 that the impact of diversity-one factor equals $p\left(\mathrm{D}_{1}\right)=(1 / 2)^{f} \simeq 3.10^{-5}$, which is negligible. Therefore, full diversity order is achievable for PARC in the observed SNR region, as shown in (13). A huge SNR gain is therefore achieved by PARC. In particular, PARC outperforms all other schemes about $5 \mathrm{~dB}$ for $N_{r}=2$ and $11 \mathrm{~dB}$ for $N_{r}=4$ at BER $=10^{-4}$.

\section{Conclusions And Discussions}

We proposed a novel cooperative scheme for a two-source multiple-relay network that combines the best relay selection and partial relaying cooperation to effectively exploit the spatial diversity. We showed by both analytical and simulation results that our proposed scheme can gain full diversity order in the finite-SNR regime when a suitable channel code is used.

The proposed scheme can easily be extended to general multi-source multi-relay networks. In this case, the selected relays might forward a number of symbols which is less than half of the codeword length. A promising application of PARC is to design a cooperation scheme to support multiple sources with different error correction capacities to achieve a given target BER. This problem can be solved by carefully designing how many symbols of each source should be relayed depending on the corresponding channel code's strength.

\section{APPENDIX A \\ Proof OF THEOREM 1}

Because the relayed symbols are randomly distributed on the codeword, the weight $d_{2}$ on the relayed block can have any integer value within $[0, d]$. Denote $\mathrm{D}_{1}=\{d, 0\}$ as the weight pattern in which all $d$ weights are not relayed. Then the weight pattern in general has one of two forms, $\mathrm{D}_{1}=\{d, 0\}$ 
and $\mathbf{D}_{d} \neq \mathrm{D}_{1}$. Using the MGF method, the UPEP can be computed as follows:

$$
\overline{\operatorname{PEP}}\left(d \mid \mathbf{D}_{d}\right)=\frac{1}{\pi} \int_{0}^{\pi / 2} \Psi_{\gamma_{\Sigma}}\left(\frac{1}{\sin ^{2} \theta}\right) d \theta .
$$

- Case 1: $\mathbf{D}_{d}=D_{1}$. In this case, all $d$ weights are located in the source-destination block, resulting in $\gamma_{\Sigma}=d \gamma_{S D}$ and $\Psi_{\gamma_{\Sigma}}(s)=\Psi_{\gamma_{S D}}(d s)$. In this case we have

$$
\begin{aligned}
\overline{\operatorname{PEP}}\left(d \mid \mathrm{D}_{1}\right) & =\frac{1}{\pi} \int_{0}^{\pi / 2} \frac{\sin ^{2} \theta}{\sin ^{2} \theta+d \bar{\gamma}_{S D}} d \theta \\
& =\frac{1}{2}\left(1-\sqrt{\frac{d \bar{\gamma}_{S D}}{1+d \bar{\gamma}_{S D}}}\right) .
\end{aligned}
$$

- Case 2: $\mathbf{D}_{d} \neq \mathrm{D}_{1}$. There is always $d_{2}$ weights are relayed, resulting in $\Psi_{\gamma_{\Sigma}}(s)=\Psi_{\gamma_{S D}}(d s) \times \Psi_{\gamma_{R^{\star}}}\left(d_{2} s\right)$. From (1) we have

$$
\begin{gathered}
\overline{\operatorname{PEP}}\left(d \mid \mathbf{D}_{d}\right)=\sum_{j=1}^{N_{r}}\left((-1)^{j-1} \sum_{\substack{n_{1}=1, \ldots, n_{j}=1 \\
n_{1} \neq \cdots \neq n_{j}}}^{N_{r}}\right. \text { (C.2) } \\
\left.\quad \frac{1}{\pi} \int_{0}^{\pi / 2} \frac{\sin ^{4} \theta}{\left(\sin ^{2} \theta+d \bar{\gamma}_{S D}\right)\left(\sin ^{2} \theta+d_{2} \bar{\gamma}_{R^{\star}, j}\right)} d \theta\right) \\
=\sum_{j=1}^{N_{r}}\left((-1)^{j-1} \sum_{\substack{n_{1}=1, \ldots, n_{j}=1 \\
n_{1} \neq \cdots \neq n_{j}}}^{N_{r}} \mathcal{I}_{1}\left(d \bar{\gamma}_{S D}, d_{2} \bar{\gamma}_{R_{i}^{\star}, j}\right)\right),
\end{gathered}
$$

where $\mathcal{I}_{1}(a, b)$ is defined in Theorem 1 , and $\bar{\gamma}_{R^{\star}, j}$ is defined in (1).

\section{APPENDIX B}

\section{PROOF OF THEOREM 2}

Let $\bar{\gamma}$ denote the referenced SNR, and $\bar{\gamma}_{S D}=G_{S D} \bar{\gamma}, \bar{\gamma}_{R^{\star}}=$ $G_{R} \bar{\gamma}$, where $G_{S D}$ and $G_{R}$ are constants depending on the network topology. We employ the upper bound of UPEP to derive diversity order for $\overline{\operatorname{PEP}}\left(d \mid \mathbf{D}_{d}\right)$ as [19]

$$
\tau \geq-\lim _{\bar{\gamma} \rightarrow \infty}\left(\frac{\log \Psi_{\gamma_{\Sigma}}(1 / 2)}{\log \bar{\gamma}}\right) .
$$

We consider two cases.

- Case 1: $\mathbf{D}_{d}=\mathrm{D}_{1}=\{d, 0\}$. There is no symbol helped by the relay and thus $\Psi_{\gamma_{\Sigma}}(1 / 2)=\Psi_{\gamma_{S D}}(d / 2)$. The diversity order in this case is given as

$$
\begin{aligned}
\tau & \geq-\lim _{\bar{\gamma} \rightarrow \infty} \frac{\log \Psi_{\gamma_{S D}}(d / 2)}{\log \bar{\gamma}} \\
& \geq-\lim _{\bar{\gamma} \rightarrow \infty}\left(\frac{\left(1+d G_{S D} \bar{\gamma} / 2\right)^{-1}}{\log \bar{\gamma}}\right)=1,
\end{aligned}
$$

which states that the UPEP has diversity order of one when $d_{2}=0$. We may write $\overline{\operatorname{PEP}}\left(d \mid \mathrm{D}_{1}\right) \propto \bar{\gamma}^{-1}$.

- Case 2: $\mathbf{D}_{d} \neq D_{1}$, then $\Psi_{\gamma_{\Sigma}}(1 / 2)=\Psi_{\gamma_{S D}}(d / 2) \times$ $\Psi_{\gamma_{R^{\star}}}\left(d_{2} / 2\right)$. Consequently, the diversity order is given as

$$
\tau \geq-\lim _{\bar{\gamma} \rightarrow \infty} \frac{\log \Psi_{\gamma_{S D}}(d / 2)}{\log \bar{\gamma}} \underbrace{-\lim _{\bar{\gamma} \rightarrow \infty} \frac{\log \Psi_{\gamma_{R^{\star}}}\left(d_{2} / 2\right)}{\log \bar{\gamma}}}_{\tau_{S e l}}
$$$$
=1+\tau_{S e l},
$$

where $\tau_{S e l}$ is the diversity order of the best relay signal (without the direct link). It is shown in [2] that the best relay selection achieves full diversity order of $N_{r}$. Therefore, we can write $\overline{\operatorname{PEP}}\left(d \mid \mathbf{D}_{d} \neq \mathrm{D}_{1}\right) \propto \bar{\gamma}^{-\left(N_{r}+1\right)}$. Combining the two cases we complete the proof of Theorem 2.

\section{REFERENCES}

[1] A. Sendonaris, E. Erkip, and B. Aazhang, "User cooperation diversity part I: System description," IEEE Trans. Commun., vol. 51, no. 11, pp. 1927-1938, 2003.

[2] A. Bletsas, A. Khisti, D. P. Reed, and A. Lippman, "A simple cooperative diversity method based on network path selection," IEEE J. Sel. Areas Commun., vol. 24, no. 3, pp. 659-672, 2006.

[3] L. Sungeun, H. Myeongsu, and H. Daesik, "Average SNR and ergodic capacity analysis for proactive and reactive DF relaying over Rayleigh fading channels," in Proc. IEEE Veh. Technol. Conf. Spring, 2009, pp. $1-5$.

[4] Y. Li, R. H. Y. Louie, and B. Vucetic, "Relay selection with network coding in two-way relay channels," IEEE Trans. Veh. Technol., vol. 59, no. 9, pp. 4489-4499, 2010.

[5] Y. Jing and H. Jafarkhani, "Single and multiple relay selection schemes and their achievable diversity orders," IEEE Trans. Wireless Commun., vol. 8, no. 3, pp. 1414-1423, 2009.

[6] S. Atapattu, Y. Jing, H. Jiang, and C. Tellambura, "Relay selection schemes and performance analysis approximations for two-way networks," IEEE Trans. Commun., vol. 61, no. 3, pp. 987-998, 2013.

[7] C. Peng, Q. Zhang, M. Zhao, Y. Yao, and W. Jia, "On the performance analysis of network-coded cooperation in wireless networks," IEEE Trans. Wireless Commun., vol. 7, no. 8, pp. 3090-3097, 2008.

[8] H. Topakkaya and W. Zhengdao, "Wireless network code design and performance analysis using diversity-multiplexing tradeoff," IEEE Trans. Commun., vol. 59, no. 2, pp. 488-496, 2011.

[9] T. Wang, A. Cano, G. B. Giannakis, and J. N. Laneman, "Highperformance cooperative demodulation with decode-and-forward relays," IEEE Trans. Commun., vol. 55, no. 7, pp. 1427-1438, 2007.

[10] A. Nasri, R. Schober, and M. Uysal, "Performance and optimization of network-coded cooperative diversity systems," IEEE Trans. Commun., vol. 61, no. 3, pp. 1111-1122, Jan. 2013.

[11] T. X. Vu, P. Duhamel, and M. Direnzo, "On the diversity of networkcoded cooperation with decode-and-forward relay selection," IEEE Trans. Wireless Commun., vol. 14, no. 8, pp. 4369-4378, Aug. 2015.

[12] X. T. Vu, M. DiRenzo, and P. Duhamel, "BER analysis of joint network/channel decoding in block Rayleigh fading channels," in Proc. IEEE Ann. Int. Symp. Personal, Indoor, and Mobile Radio Commun., London, 2013, pp. 698-702.

[13] A. Eckford, J. Chu, and R. Adve, "Low complexity and fractional coded cooperation for wireless networks," IEEE Trans. Wireless Commun., vol. 7, no. 5, pp. 1917-1929, May 2008.

[14] M. Khormuji and E. Larsson, "Cooperative transmission based on decode-and-forward relaying with partial repetition coding," IEEE Trans. Wireless Commun., vol. 8, no. 4, pp. 1716-1725, Apr. 2009.

[15] L. Zheng and D.N.C. Tse, "Diversity and Multiplexing: A Fundamental Tradeoff in Multiple-Antenna Channels", IEEE Trans. Inf. Theory, vol. 49, no. 5, May 2003.

[16] T. Cover and A. E. Gamal, "Capacity theorems for the relay channel," IEEE Trans. Inf. Theory, vol. 25, no. 5, pp. 572-584, May 1979.

[17] R. Narasimhan, "Finite-SNR DiversityMultiplexing Tradeoff for Correlated Rayleigh and Rician MIMO Channels", IEEE Trans. Inf. Theory, vol. 52, no. 9, Sept. 2006.

[18] L. Bahl, J. Cocke, F. Jelinek, and J. Raviv, "Optimal decoding of linear codes for minimizing symbol error rate (corresp.)," IEEE Trans. Inf. Theory, vol. 20, no. 2, pp. 284-287, 1974.

[19] M. K. Simon and M.-S. Alouini, Digital communication over fading channels, 2nd ed., ser. Wiley series in telecommunications and signal processing. Hoboken, N.J.: John Wiley \& Sons, 2005.

[20] A. Glavieux, Channel Coding in Communication Networks: From Theory to Turbo Codes. Wiley\& Sons Ltd, 2007.

[21] T. X. Vu, N. Q. B. Vo, M. Di Renzo, and P. Duhamel, "Performance analysis of relay networks with channel code in low SNR regime," in Proc. IEEE Int. Workshop Signal Process. Adv. Wireless Commun., Darmstadt, 2013, pp. 575-579.

[22] P. Frenger, P. Orten, and T. Ottosson, "Convolutional codes with optimum distance spectrum," IEEE Commun. Lett., vol. 3, no. 11, pp. 317-319, 1999 